\title{
When the brain simulates stopping: Neural activity recorded during real and imagined stop-signal tasks
}

\author{
Alberto J. González-Villar ${ }^{1}$ • F. Mauricio Bonilla ${ }^{2}$ • María T. Carrillo-de-la-Peña ${ }^{1}$
}

Published online: 9 May 2016

(C) Psychonomic Society, Inc. 2016

\begin{abstract}
It has been suggested that mental rehearsal activates brain areas similar to those activated by real performance. Although inhibition is a key function of human behavior, there are no previous reports of brain activity during imagined response cancellation. We analyzed event-related potentials (ERPs) and time-frequency data associated with motor execution and inhibition during real and imagined performance of a stop-signal task. The ERPs characteristic of stop trials- that is, the stop-N2 and stop-P3 - were also observed during covert performance of the task. Imagined stop (IS) trials yielded smaller stop-N2 amplitudes than did successful stop (SS) and unsuccessful stop (US) trials, but midfrontal theta power similar to that in SS trials. The stop-P3 amplitude for IS was intermediate between those observed for SS and US. The results may be explained by the absence of error-processing and correction processes during imagined performance. For go trials, real execution was associated with higher mu and beta desynchronization over motor areas, which confirms previous reports of lower motor activation during imagined execution and also with larger P3b amplitudes, probably indicating increased top-down attention to the real task. The similar patterns of activity observed for imagined and real performance suggest that imagination tasks may be useful for training inhibitory processes. Nevertheless, brain activation was generally weaker during mental rehearsal, probably as a result of the
\end{abstract}

Alberto J. González-Villar

albertojac.gonzalez@gmail.com

1 Clinical Psychology and Psychobiology, Faculty of Psychology, University of Santiago de Compostela, 15782 Santiago de Compostela, Spain

2 Laboratory of Experimental Psychology, Faculty of Psychology, University El Bosque, Bogotá, Colombia reduced engagement of top-down mechanisms and limited error processing.

Keywords Motor inhibition · Stop-signal task · Mental rehearsal $\cdot$ Event-related potentials $\cdot$ Time-frequency analysis

Inhibition is the ability to suppress, withhold, or interrupt ongoing and planned actions in response to changes in the environment or in our own impulses, and is crucial for controlling cognitive and motor events (Ramautar, Kok, \& Ridderinkhof, 2006). Enhanced inhibitory ability has been related to better performance in several sports (Alves et al., 2013; Verburgh, Scherder, van Lange, \& Oosterlaan, 2014; Wang et al., 2013), whereas deficits in this function can lead to the execution of inappropriate responses and are usually associated with disorders such as hyperactivity or addictive and compulsive behavior (Chamberlain, Fineberg, Blackwell, Robbins, \& Sahakian, 2006; Lawrence, Luty, Bogdan, Sahakian, \& Clark, 2009; Lijffijt, Kenemans, Verbaten, \& van Engeland, 2005).

Mental practice implies rehearsal of an action without execution of any overt physical movement and may be useful for enhancing performance when active movement training is not feasible (Driskell, Copper, \& Moran, 1994). It has been suggested that neuronal changes in motor networks also occur during mental rehearsal, providing an argument for the effectiveness of combined physical and mental practice (Cisek \& Kalaska, 2004). The functional equivalence and neural overlapping of action execution and action simulation have also been suggested (Carrillo-de-la-Peña, Galdo-Álvarez, \& Lastra-Barreira, 2008; Galdo-Álvarez \& Carrillo-de-la-Peña, 2004; Grèzes \& Decety, 2001; Jeannerod \& Frak, 1999). Although covert training in inhibitory skills may be useful for treating several deficits, especially when an overt response is not feasible, the brain activity associated with imagined 
inhibition has not yet been explored. In the present study, we investigated the brain electrical activity associated with real and imagined movement execution and movement cancellation occurring during the performance of a stop-signal task. Given the increasing use of brain-computer interfaces, study of the neural activity associated with imagined performance of inhibition may also be of interest for developing new communicating systems for such devices.

The stop-signal task is one of the main paradigms for measuring inhibitory control. It consists of the presentation of a stream of "go" stimuli that request execution of an action (e.g., pressing a button), and some of the "go" signals are followed by a "stop" signal, indicating that the action must be cancelled. Given the delayed presentation of the "stop" signal, some of the processes involved in the execution of the response have already started, and therefore an inhibitory reactive response is required for correct performance. Thus, this experimental paradigm requires greater inhibitory pressure on response-related processes than do other inhibition tasks, such as the go/no-go task, in which participants must respond quickly to most of the stimuli and withhold the response to the remaining ones (Aron, 2011; Kok, Ramautar, De Ruiter, Band, \& Ridderinkhof, 2004). The horse-race model illustrates the factors mediating inhibitory performance. According to this model, the processes involved in the go response race against the processes associated with the stop response. If the go response finishes the race first, it will be executed; on the contrary, if the stop response manages to rectify the go response prior to its execution, the outcome will be a successful inhibition (Band, van der Molen, \& Logan, 2003; Logan, 1994).

Electroencephalographic (EEG) recording is a valuable tool for investigating the neural processes involved in response inhibition, because it does not require any overt response to observe modulations and has excellent temporal resolution. Event-related potentials (ERPs) provide detailed information about the moment at which the brain detects the stop signal and "decides" to stop ongoing actions. Because ERPs can be distorted if the latencies of the components vary across trials, thus reducing or cancelling their average amplitudes (Müller \& Anokhin, 2012; Schmiedt-Fehr \& BasarEroglu, 2011), the complementary use of time-frequency analysis provides additional information about the power of each frequency at each time point.

In the psychophysiological study of inhibition, one of the most characteristic ERPs is the stop-N2, a fronto-central negative wave that peaks at around 200-300 ms after a stop signal. Although there is some controversy about the functional significance of this potential, it is mainly thought to reflect conflict monitoring (Huster, Enriquez-Geppert, Lavallee, Falkenstein, \& Herrmann, 2013). The amplitude of the stop-N2 increases with differences in the actual and desired responses, even without the need for response inhibition (Enriquez-Geppert, Konrad, Pantev, \& Huster, 2010; Randall \& Smith, 2011). This wave is usually modulated by correctly or incorrectly inhibited responses, with larger amplitudes for unsuccessfully stopped (US) than for successfully stopped (SS) responses (Dimoska, Johnstone, \& Barry, 2006; Kok et al., 2004; Ramautar et al., 2006). Time-frequency data have shown that the N2 is associated with theta oscillations (around 4-7 Hz) over fronto-central scalp areas, an activity also related to conflict detection and error processing (Harmony, Alba, Marroquín, \& GonzálezFrankenberger, 2009; Harper, Malone, \& Bernat, 2014; Kirmizi-Alsan et al., 2006; Luu, Tucker, \& Makeig, 2004; Müller \& Anokhin, 2012). The neural generator of the N2 is presumed to be located around the dorsal portion of the anterior cingulate cortex (Botvinick, Cohen, \& Carter, 2004; Braver, Barch, Gray, Molfese, \& Snyder, 2001; Nieuwenhuis, Yeung, van den Wildenberg, \& Ridderinkhof, 2003).

Another classical ERP evoked with the stop-signal task is the stop-P3, a P3a-like wave with maximum amplitude at central electrodes. Although this potential was initially interpreted as motor inhibition (Enriquez-Geppert et al., 2010; Randall \& Smith, 2011), given its delayed latency with respect to reaction times (RTs), it is now viewed as the cognitive evaluation of this motor inhibition (Huster et al., 2013). Contrary to the stop-N2, the amplitude of the stop-P3 is usually higher in SS than in US trials (Dimoska et al., 2006). The onset of this component is highly correlated with the RT of stopping, so it is a good indicator of response inhibition (Wessel \& Aron, 2015), although it seems to be dependent on earlier processes of inhibition (Huster, Plis, Lavallee, Calhoun, \& Herrmann, 2014). Several neural sources, such as the mid-cingulate cortex and the inferior frontal cortex, appear to be involved in generating the stop-P3 wave (e.g., Huster, Westerhausen, Pantev, \& Konrad, 2010; Rubia, Smith, Taylor, \& Brammer, 2007; Schall, Stuphorn, \& Brown, 2002).

The main objective of the present study was to test whether imagined performance of the stop-signal task evokes a pattern of brain electrical activity associated with stop trials similar to that observed during overt performance of the task. In this report, we used sounds as the stop signals, a modality that has been associated with faster inhibitory responses than visual stop signals (Ramautar et al., 2006). Because neural activity may be modulated by the outcome of the inhibition, we separated SS and US trials, and compared both with imagined stop (IS) trials. To better clarify the possible differences across conditions and to analyze the EEG activity specifically related to inhibition (ruling out the contribution of exogenous potentials associated with processing of the go and stop signals), we applied Laplacian spatial filtering and temporal principal component analyses (tPCA) to identify the main components, and we also performed complementary time-frequency analyses.

We expected that covert performance of the stop-signal task would produce a pattern of EEG activity similar to that found during the task's real execution, including stop-N2 and stop-P3. We also hypothesized that the brain activity should 
be different for failed and correct inhibition, thus producing larger stop-N2 amplitude and midfrontal theta power for US, and a larger stop-P3 for SS, as has previously been reported. We also expected lower conflict levels and less inhibitory pressure in IS trials, which should be reflected in smaller stop-N2 and stop-P3 amplitudes and theta power around midfrontal locations.

A second aim was to replicate previous data on the functional equivalence between action execution and motor imagery, by comparing ERPs and time-frequency data obtained from go trials across performance conditions. We expected to confirm previous findings about $\mathrm{mu}(9-13 \mathrm{~Hz})$ and beta (18-24 Hz) power reductions over the Rolandic areas during both motor performance and imagery (McFarland, Miner, Vaughan, \& Wolpaw, 2000; Pfurtscheller \& Neuper, 1997). To unravel the possible role of attention in the differences between conditions, we also studied the go-P3 - a parietal positivity peaking around $300 \mathrm{~ms}$, the amplitude of which is modulated by attention levels or task demands (Kok, 2001).

\section{Method}

\section{Participants}

Twenty-six healthy volunteers (12 women, 14 men) were recruited from the University of Santiago de Compostela: 23 were undergraduate students, and three were postgraduate students. The ages of the volunteers ranged from 20 to 38 years (mean = 22.8 years, $S D=4.9$ years). All had normal or corrected-tonormal vision and were right-handed, according to the Edinburgh Handedness Inventory. None of them had any history of neurological or psychiatric disorders, nor drug abuse. The data from two participants were rejected because of problems during the EEG recording and insufficient numbers of trials.

We obtained approval from the University of Santiago de Compostela Research Ethics Committee before undertaking the project.

\section{Stimuli and apparatus}

Participants were seated comfortably on an armchair in a dimly lit, sound-attenuated room. They were instructed to fix their gaze on a plus sign that was projected on a computer screen during execution of the task. The stop task was conducted in real and imagined modes. Arrows were presented in the center of the screen pointing to the left or the right side, with a duration of $500 \mathrm{~ms}$ and an interstimulus interval of $2 \mathrm{~s}$. Participants were instructed to either press or imagine pressing a button with their right or left thumb, in response to the direction of the arrow. In the unpredictable and infrequent stop trials, the arrows were followed by a sound $(1000 \mathrm{~Hz}$ and $80 \mathrm{~dB}$ ) signaling that the motor responses had to be stopped.
In the real condition, the time interval between the gosignal and stop-signal onsets started at $300 \mathrm{~ms}$, although this varied during the task, depending on the participant's performance (ranging from 160 to $400 \mathrm{~ms}$, with 40 -ms steps). A staircase tracking algorithm was used to adjust the interval between the go and stop signals in some trials, depending on the results of the previous stop trial (Band \& van Boxtel, 1999). This algorithm yields around half successful and half unsuccessful response-inhibited trials. If the response in the previous stop trial was correctly inhibited, the interval between the go and stop signals in the next stop trial was longer, also increasing the difficulty of successful inhibition; if the participant had made a response in the previous stop trial, the interval between the signals in the next stop trial was shorter (Logan \& Cowan, 1984).

In the motor imagery condition, participants were instructed to imagine as vividly as possible responding with the hand on the same side indicated by the arrow, and withholding the response when they heard the sound. They had to keep their hands on the response box. In this condition, the stop signal was always presented $300 \mathrm{~ms}$ after the go signal.

Each task consisted of 280 trials: $70 \%$ go and $30 \%$ stop. The total number of go trials was 196 (98 for each direction). The total number of stop trials was 84 (42 for each direction). The task was designed and presented with the STIM program (Neuroscans Labs) on a 15-in. screen at a distance of $1 \mathrm{~m}$ from the participant. Participants completed some practice trials before the real-condition task. The order of the tasks was always the same: first overt execution, and then covert performance. This procedure was used to ensure more effective mental rehearsal after real practice. Participants were allowed a 5-min rest between the two tasks.

\section{Psychophysiological recording}

EEG recordings were obtained from $28 \mathrm{Ag} / \mathrm{AgCl}$ scalp electrodes (10-20 International System) inserted in an electrode cap (ElectroCap International) and referenced to the left and right mastoids. Four additional surface electrodes placed $1 \mathrm{~cm}$ above and below and in the canthus of the eyes were used to monitor ocular movements. The AFz electrode was used as the ground. Impedance was maintained below $10 \mathrm{k} \Omega$. The EEG signal was sampled at $500 \mathrm{~Hz}$ and amplified 10,000 times (using a Synamp Neuroscan amplifier) before being filtered with a 0.1 - to $100-\mathrm{Hz}$ online band-pass filter and a $50-\mathrm{Hz}$ notch filter. For exclusion of trials with muscle activity during the imagination task, electromyogram (EMG) activity was recorded with two cup electrodes (1-cm diameter) on the ventral side of each forearm, trisecting the wrist-elbow distance, to record the activity of the flexor digitorum profundis, the flexor digitorum superficialis, and the flexor pollicis longus of both arms. 


\section{Offline processing}

The EEG data were analyzed using the EEGlab 12.02 toolbox (Delorme \& Makeig, 2004). The data were resampled to $250 \mathrm{~Hz}$ and re-referenced to an average reference. The data from poorly recording channels were replaced using spherical-spline interpolation. EEG segments containing large ocular or other artifacts were rejected after visual inspection. The data were filtered using a low-pass $30-\mathrm{Hz}$ FIR filter. Then 3 -s epochs were extracted, from $1 \mathrm{~s}$ prestimulus to $2 \mathrm{~s}$ poststimulus. For extraction, go epochs were time-locked to the go stimuli (arrows), and stop epochs were time-locked to the stop stimuli (tones). An independent component analysis algorithm was applied to remove components related to ocular artifacts. We also performed a current source density transformation based on the spherical-spline surface Laplacian, using the MATLAB code provided by Kayser and Tenke (2006) (smoothing constant $=10^{-5}$ ). This type of filter removes spatially broad features of the data while leaving local features unmodified, and produces sharper topographies of the ERPs and time-frequency modulations, while reducing their overlap due to volume conduction. The ERPs obtained were baseline corrected from -200 to $0 \mathrm{~ms}$.

Interpretation of the EEG data in a stop-signal task is complicated by the overlap between the electrical activity associated with the go signal and that associated with the following stop stimulus. To solve this issue, time-shifted go trials were subtracted from the stop trials in each participant using the following procedure. Go trials with the fastest RTs were subtracted from US trials, whereas trials with the slowest RTs were subtracted from SS trials. For this purpose, the proportions of US and SS trials were first calculated for each stop signal delay $(\mathrm{SSDs}=160,200,240,280,320,360$, or $400 \mathrm{~ms}$ ). Go epochs were then divided into fast go and slow go epochs on the basis of this proportion and were averaged; that is, if the proportion of US trials for each SSD was .55, the fastest $55 \%$ of go trials were selected to apply the US-minusgo correction, whereas the slowest $45 \%$ were used for the SSminus-go correction. The fast-go average was then multiplied by the proportion [trials in the current SSD/total number of US trials], shifted in time to match the current SSD and then subtracted from the US epochs. The same procedure was applied to the SS trials, but using the slow-go epochs. For IS, at a fixed SSD of $300 \mathrm{~ms}$, the go ERP was averaged, shifted $300 \mathrm{~ms}$, and then subtracted from the IS epoch. This procedure enabled comparison of the stop trials while restricting the influence of the go signal. Similar procedures were applied elsewhere (Kok et al., 2004; Krämer, Knight, \& Münte, 2011; Krämer et al., 2013; Ramautar et al., 2006).

To avoid including data on real hand movements during the IS task, we calculated the root mean square of the EMG data. Epochs in which the maximum value of the root mean squared EMG data (from 100 to $1,000 \mathrm{~ms}$ poststimulus) was more than twice the maximum point at baseline (from -400 to $-100 \mathrm{~ms}$ ) were rejected. Figures $1 \mathrm{c}$ and $2 \mathrm{c}$ show the rectified EMG activity for the stop and go trials, respectively. The rectified EMG was obtained by high-pass filtering the signal at $20 \mathrm{~Hz}$; all time points were then converted to absolute values and finally low-pass filtered at $50 \mathrm{~Hz}$. The final number of trials for each condition was as follows: go real, mean $=182, S D=$ 8; go imagined, mean $=189, S D=5 ; \mathrm{SS}$, mean $=46, S D=10$; $\mathrm{US}$, mean $=37, S D=10$; and $\mathrm{IS}$, mean $=80, S D=2$.

Principal component analysis After ERP averaging, we found that the stop-N2 appeared to overlap with the auditory N1 evoked by the stop tone (see Fig. 1a). To solve this problem - in addition to the previously commented Laplacian spatial filter - we applied a tPCA to the ERP data by using the ERP PCA toolkit (Dien, 2010). The tPCA enables temporally overlapping components to be separated (Dien \& Frishkoff, 2005). The combination of a Laplacian filter and tPCA has been described in depth by Kayser and Tenke (2006). A scree test was used to select from among the eight temporal factors (TFs) extracted using the covariance matrix (Promax rotation). Three of the TFs were identified as specific components because of their latency, polarity, and topography: TF6 (which peaked at $108 \mathrm{~ms}$ in $\mathrm{C} 3 / \mathrm{C} 4$ and explained $7.5 \%$ of the variance) was identified as the N1; TF7 (which peaked at $152 \mathrm{~ms}$ in FCz and explained 7.2\% of the variance) was identified as the stop-N2; and TF1 (which peaked at $228 \mathrm{~ms}$ in $\mathrm{Cz}$ and explained $16 \%$ of the variance) was identified as the stopP3. The remaining factors were not included in the analyses because they were not related to the components of interest, given their latencies and topographies. See Fig. $1 \mathrm{~b}$.

Factor loadings, reconstructed to microvolts per square centimeter $\left(\mu \mathrm{V} / \mathrm{cm}^{2}\right)$ and measured at the electrode at which each factor peaked, were analyzed for every participant and condition (SS, US, and IS).

The tPCA was also applied to go trials, with the same parameters. In this case, we extracted eight TFs and identified one factor as the $\mathrm{P} 3 \mathrm{~b}$ (TF1, with a peak at $396 \mathrm{~ms}$ in $\mathrm{Pz}$ electrode, explaining $24 \%$ of the variance) (see Fig. 2b). Differences in the factor loadings between real versus imagined conditions were analyzed.

Time-frequency analyses Time-frequency analyses were performed by convolving the EEG data with a family of complex Morlet wavelets that ranged in frequency from 3 to $30 \mathrm{~Hz}$ in 20 logarithmically increasing steps, and with logarithmically increasing cycles, from three cycles at the lowest frequency to eight at the highest frequency. The power data obtained after convolution were transformed by calculating the common logarithm $\left(\log _{10}\right)$. This procedure was applied to reduce the deviation of the original power values and to obtain easierto-handle data. In stop trials, the same correction procedure applied to the ERPs was used. In this case, the log- 

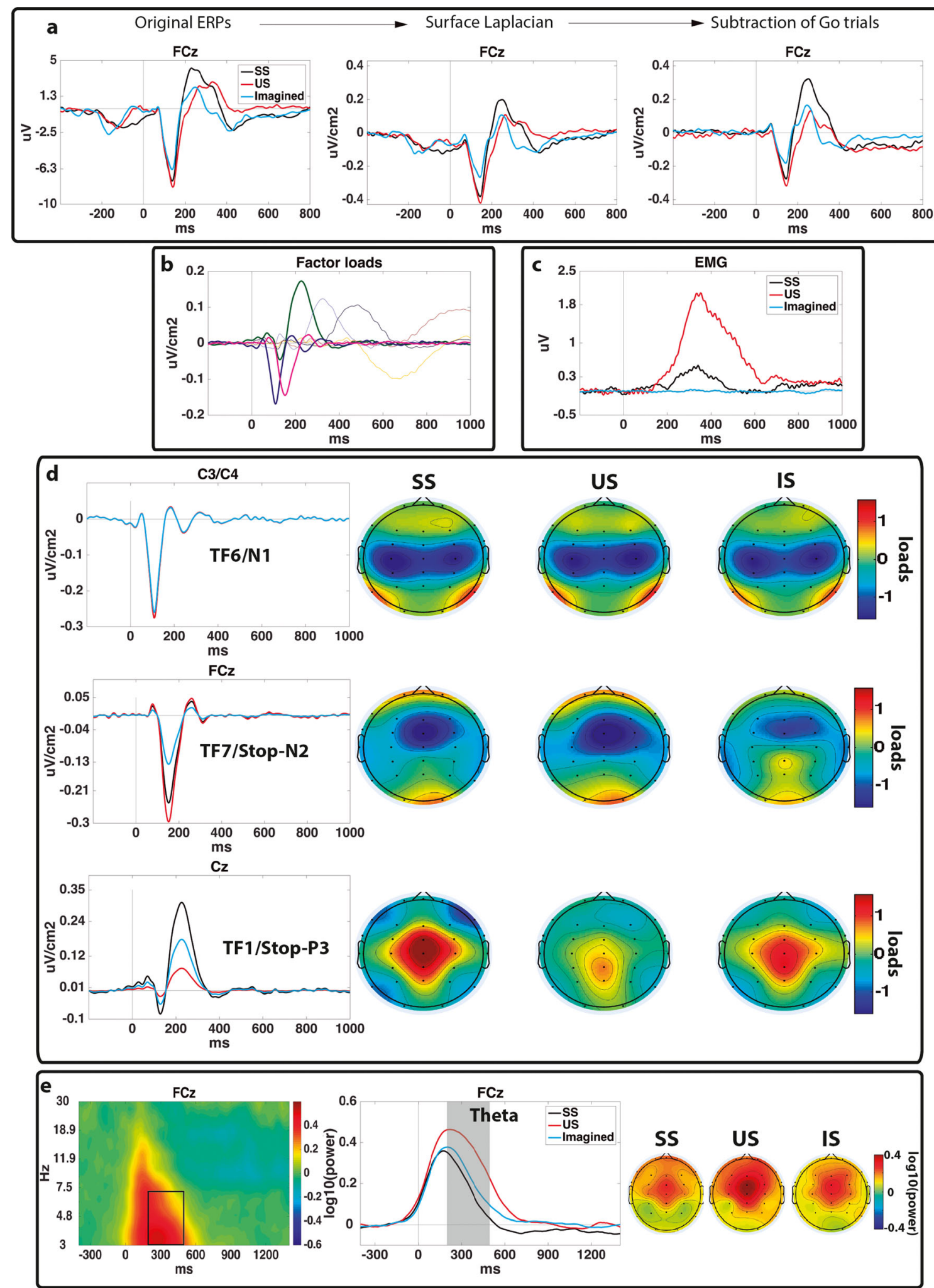

Fig. 1 (a) Event-related potential (ERP) grand averages for stop trials at the $\mathrm{FCz}$ electrode in the original form, after spatial filtering, and after subtraction of go activity. (b) Temporal principal components analysis (tPCA) decomposition for stop trials. The selected factors are highlighted in bold type. (c) Rectified electromyography for each condition. (d)
Waveforms of the selected tPCA factors (TF6[N1], TF7[stop-N2], and TF1[stop-P3]) and topographies for each stop condition. (e) Left: Average power spectrograms for all conditions. Middle: Time courses of theta power. Right: Topographies of theta power (between 200 and $500 \mathrm{~ms}$ and 3 and $7 \mathrm{~Hz}$ ) 

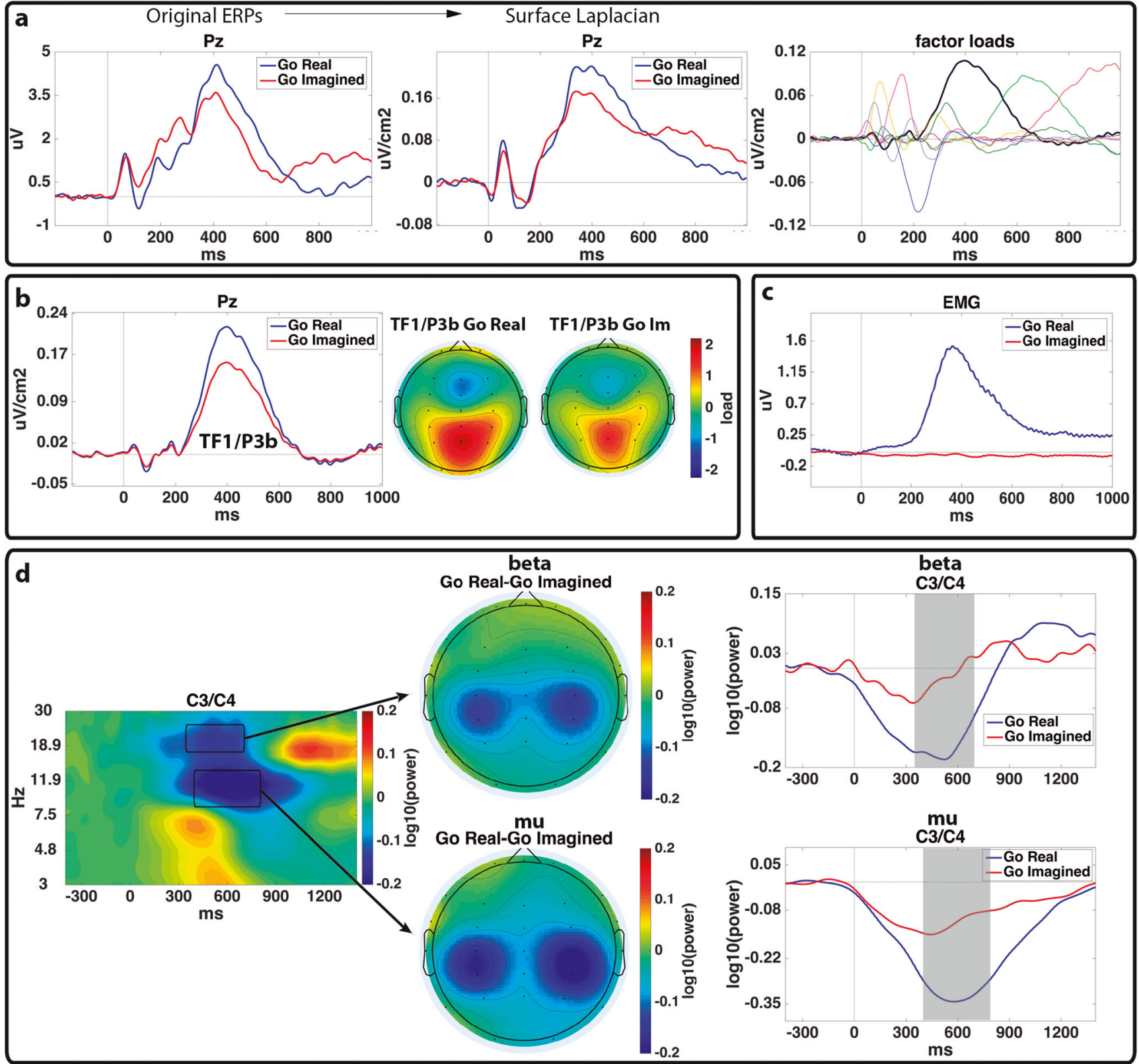

Fig. 2 (a) Left: ERP grand averages for go trials in the real and imagined conditions registered at the Pz electrode. Middle: ERPs after Laplacian filtering. Right: tPCA decompositions for go trials (the selected factor is highlighted in bold type). (b) Reconstructed waveforms of the TF1[go$\mathrm{P} 3]$ at the $\mathrm{Pz}$ electrode and the topographies in real and imagined go conditions. (c) Rectified electromyography for each condition. (d) Left:

transformed power values of go trials were subtracted from the log-transformed power for stop trials at each frequency, and the time delay between go and stop trials was corrected. Finally, baseline correction was applied by subtracting the average baseline period (from -400 to $-100 \mathrm{~ms}$ ) for each frequency.

Time-frequency windows were selected for further analyses after averaging all conditions together (go real and go imagined for go; SS, US, and IS for stop). Values of the
Average power spectrograms for both conditions. Boxes show the frequencies (beta and mu) and latencies analyzed by Student's $t$ tests. Middle: Topographies of the go real - go imagined differences calculated using the mean values from the boxes. Right: Time courses of mu and beta power. The shaded areas were submitted to statistical comparisons

windows with higher power modulations were averaged for statistical comparison. This procedure was used to avoid making assumptions about the potential differences among the conditions. A window from 200 to $500 \mathrm{~ms}$ and 3 to $7 \mathrm{~Hz}$ was selected at the $\mathrm{FCz}$ electrode for testing the midfrontal theta activity on stop trials. We extracted the mean power from 400 to $800 \mathrm{~ms}$ and 9 to $13 \mathrm{~Hz}$ for the mu oscillations in $\mathrm{C} 3 / \mathrm{C} 4$, and from 350 to $700 \mathrm{~ms}$ and 18 to $24 \mathrm{~Hz}$ in $\mathrm{C} 3 / \mathrm{C} 4$ for the beta band, only in go trials. 


\section{Statistical analyses}

For stop trials, we used one-way repeated measures analyses of variance (ANOVAs) to compare the SS, US, and IS trials, for each potential (N1, stop-N2, and stop-P3) and theta power. For go trials, we used paired $t$ tests to compare the real and imagined conditions in terms of $\mathrm{P} 3 \mathrm{~b}$ and mu and beta activity. We applied Greenhouse-Geisser corrections to control for sphericity violation, when appropriate. We used the Shapiro-Wilk test to verify the normality of all measures.

\section{Results}

\section{Stop trials}

The proportion of SS trials was $56.3 \%(S D=16 \%)$, and that of US trials was $43.7 \%(S D=16 \%)$. The mean RT for US trials was $122 \mathrm{~ms}(S D=87 \mathrm{~ms})$ after appearance of the stop signal. The mean SSDs were $252 \mathrm{~ms}(S D=32 \mathrm{~ms})$ for the real task, $245 \mathrm{~ms}(S D=37 \mathrm{~ms})$ for SS, and $260 \mathrm{~ms}(S D=31 \mathrm{~ms})$ for US trials. The estimated stop-signal RT was $138 \mathrm{~ms}$ $(S D=32 \mathrm{~ms}){ }^{1}$

Table 1 shows the mean amplitudes for tPCA factors and theta power for each condition. A similar pattern of brain electrical activity was obtained during stop trials in the real and imagined task (Fig. 1).

The repeated measures ANOVAs with Condition (US, SS, and IS) as a between-subjects factor produced the following results:

TF6 (N1): No differences were observed in the TF6-N1 factor $[F(1.5,34.4)=0.79, p=.45]$.

TF7 (stop-N2): A main effect of condition $[F(1.6,36.3)=$ $12.22, p<.001]$ was observed; post-hoc comparisons showed differences between SS and IS $(p=.007)$ and between US and IS ( $p=.002)$, with no significant difference between SS and US $(p=.16)$. See Table 1 .

TF1 (stop-P3): A significant main effect was observed for this factor $[F(1.6,36.7)=18.88, p<.001]$. Post-hoc comparisons revealed differences between SS and US $(p<.001)$, between US and IS $(p=.005)$, and between SS and IS $(p=.006)$.

Differences were also observed in midfrontal theta power $[F(2,46)=23.6, p<.001]$. Post-hoc comparisons showed differences between US and both SS $(p=.001)$ and IS $(p<.001)$ conditions, but not between SS and IS trials $(p=.89)$ (see Fig. 1e).

\footnotetext{
${ }^{1}$ The stop-signal RT was estimated using a variant of the integration method (see van den Wildenberg, van der Molen, \& Logan, 2002, and Verbruggen \& Logan, 2009, for detailed descriptions).
}

\section{Go trials}

Participants responded correctly to $97.4 \%(S D=3.6 \%)$ of the go trials in the real condition. The mean RT for correct go trials was $423 \mathrm{~ms}(S D=71 \mathrm{~ms})$.

Student's $t$ tests produced the following results:

TF1 (go-P3) had a higher peak amplitude for the real (mean $=0.22 \mu \mathrm{V} / \mathrm{cm}^{2}, S D=0.14$ ) than for the imagined (mean $\left.=0.16 \mu \mathrm{V} / \mathrm{cm}^{2}, S D=0.11\right)$ condition, $[t(23)=3.5$, $p=.002]$. See Fig. 2 b.

In the time-frequency data, differences were observed over motor areas in mu power, with higher desynchronization for real (mean $=-0.3, S D=0.18$ ) than for imagined (mean $=-0.11, S D=0.13)$ go trials $[t(23)=-4.48, p<.001]$. Similarly to mu oscillations, differences were observed over motor areas in the beta band, with greater desynchronization for real (mean $=-0.15, S D=0.1)$ than for imagined (mean = $-.03, S D=.06)$ go trials $[t(23)=5.7, p<.001]$. See Fig. $2 \mathrm{~d}$.

\section{Discussion}

The ability to inhibit erroneous or unwanted responses is crucial in everyday life and can potentially be improved by mental rehearsal. The main goal of the present study was to analyze the brain electrical activity associated with performance of an inhibitory stop-signal task during real and imagined execution. To better understand the functional significance of ERP indices of inhibition and the temporal dynamics of the EEG, the analyses included spatial filtering, tPCA, and time-frequency decomposition. Overall, the results support the idea that similar brain electrical activity occurs during imagined motor inhibition and real performance.

A limitation of using the stop-signal task to investigate inhibitory mechanisms is that the EEG of stop trials include neural activity elicited by both the go stimuli and the stop signal. Although the influence of the go signal is removed from the stop trials, it is difficult to dissociate the neural activity associated with processing of the perceptual cue (the stop signal itself) from that linked to response inhibition. In the present study, two different strategies were used to overcome these limitations. Go-related activity was removed from stop-trial activity; in the case of real performance, differences in stop signal delays and successful versus unsuccessful stopping were taken into account to calculate the difference waves. In order to avoid overlap between the ERP exogenous potentials and indices of inhibition, Laplacian spatial filtering and tPCA decomposition were then applied. This is especially useful when taking into account that we used stop signals in the auditory mode, which have been shown to cause overlap between the auditory N1 and the inhibitory stop-N2 (Bekker, Kenemans, Hoeksma, Talsma, \& Verbaten, 2005; Dimoska \& Johnstone, 2007; Hughes, Fulham, Johnston, \& Michie, 2012; 
Table 1 Peak amplitudes of tPCA factors and theta power for each stop condition

\begin{tabular}{|c|c|c|c|c|}
\hline Condition $\backslash$ Factor & TF6 (N1) & TF7 (Stop-N2) & TF1 (Stop-P3) & Theta \\
\hline Successfully stopped & $-0.25(0.11)$ & $-0.24(0.19)$ & $0.30(0.29)$ & $0.23(0.16)$ \\
\hline Unsuccessfully stopped & $-0.25(0.11)$ & $-0.30(0.23)$ & $0.08(0.21)$ & $0.42(0.13)$ \\
\hline Imagined stopped & $-0.24(0.11)$ & $-0.14(0.14)$ & $0.18(0.18)$ & $0.27(0.11)$ \\
\hline
\end{tabular}

Factor amplitudes are reported in $\mu \mathrm{V} / \mathrm{cm}^{2}$. The final column includes power values for theta oscillations at the FCz electrode. Standard deviations are shown in parentheses

Lansbergen, Böcker, Bekker, \& Kenemans, 2007; Ramautar et al., 2006).

The tPCA also clearly identified a fronto-central stop-N2 factor and a later stop-P3 factor peaking at $\mathrm{Cz}$ during covert performance of the stop-signal task.

The amplitude of the stop-N2 was significantly smaller during imagined than during real stopping, whereas no differences between SS and US were observed. It is possible that the results may be explained by differences in the levels of attention across conditions. Our data indicated that stop trials were processed similarly in US, SS, and even IS trials, in contrast to the findings of Bekker et al. (2005), who reported larger auditory N1 amplitudes for SS than for US trials, suggesting that successful inhibition partly depends on the level of attention. These results rule out the contribution of exogenous ERPs in the observed differences between conditions.

Although there is some controversy about its functional significance, the midfrontal stop-N2 is often related to conflict monitoring (Botvinick et al., 2004; Huster et al., 2013). It appears to belong to a family of other midfrontal negative ERPs that are evoked after events that require realization of the need for control (see Cavanagh \& Frank 2014). On the basis of previous reports, we hypothesized that the amplitude of the stop-N2 would be larger during unsuccessful stopping. Although the mean N2 amplitude was larger for US trials, the difference between US and SS trials was far from significant. Similar results have been reported by other authors (Pliszka, Liotti, \& Woldorff, 2000; Schmajuk, Liotti, Busse, \& Woldorff, 2006; Shen, Lee, \& Chen, 2014). We further analyzed the original ERPs and found that the difference between SS and US trials was also not significant $(p=.095)$, indicating that the procedures applied (Laplacian filter, stop-minus-go ERPs, and PCA) were not critical for this result.

Given that the difference in stop-N2 amplitudes between overt and covert performance does not seem to depend on bottom-up processing of the stop signal, as was shown by the similar N1 amplitudes, our data suggest differences in further processing steps. This finding can probably be explained by topdown mechanisms implied in conflict monitoring and related to activity around medial-frontal brain locations (Botvinick et al., 2004; Braver et al., 2001; Nieuwenhuis et al., 2003). To clarify this point, we analyzed midfrontal theta power and found no difference between IS and SS, both of which produced significantly smaller theta power than US trials. The results of time-frequency analyses therefore suggest that the level of conflict during IS trials may be similar to that in SS trials, in which no error is present, but is much lower than for failed inhibition. These results are consistent with the view of this oscillatory activity as the realization of the need for control (Cavanagh, Zambrano-Vazquez, \& Allen, 2012), which should be higher for trials with incorrect responses. In addition, they may reflect the presence, in the US condition, of the error-related negativity (ERN), a negative potential that appears around 50-60 ms after an erroneous response (Dehaene, Posner, \& Tucker, 1994), which is mainly generated by the midfrontal theta that is phase-locked to the response (Luu et al., 2004).

We identified a positive tPCA factor peaking at the $\mathrm{Cz}$ electrode as the stop-P3, the amplitude of which was higher for SS than for US trials, as has been previously reported (Dimoska et al., 2006; Kok et al., 2004). In the imagined condition, the stopP3 was also larger than in US, but smaller than in SS, trials. Although it has been assumed that the stop-P3 represents motor inhibition (Enriquez-Geppert et al., 2010; Randall \& Smith, 2011), this wave has recently been associated with the evaluation of inhibitory performance and adaptation to the inhibitory demands, rather than with pure motor inhibition (Huster et al., 2013). Our results are consistent with this interpretation. We found stop-P3 latencies longer than the US RTs. The larger stop-P3 amplitudes for SS than for US trials also support the relation between the stop-P3 and the outcome of the inhibition (Dimoska et al., 2006; Ramautar et al., 2006). The differences between successful and failed trials may be caused by the detection of errors in the latter; thus, the overlapping of theta oscillations (and the ERN) may explain the reported reduction in the stop-P3 amplitude during failed inhibition.

A second goal of the present study was to replicate previous data on the functional equivalence between action execution and motor imagery. Previous reports with the lateralized readiness potential (LRP) paradigm suggested that similar patterns of primary motor cortex activation are present in overt and covert actions, although with diminished amplitude in the latter (Galdo-Álvarez \& Carrillo-de-la-Peña, 2004; Carrillo-de-laPeña, Lastra-Barreira, \& Galdo-Álvarez, 2006; Kranczioch, Mathews, Dean, \& Sterr, 2009). Mu and beta depletion over Rolandic areas is a well-known correlate of motor execution. Consistent with the LRP data, the same pattern of mu and beta 
reductions has been found during motor imagery and actual movement (Pfurtscheller \& Neuper, 1997; Pfurtscheller, Neuper, Brunner, \& da Silva, 2005; Pfurtscheller, Brunner, Schlögl, \& Lopes da Silva, 2006; Stavrinou, Moraru, Cimponeriu, Della Penna, \& Bezerianos, 2007).

Our analysis of the motor-related EEG in go trials revealed significantly greater reduction of $\mathrm{mu}$ and beta power for real than for imagined go trials. This finding is consistent with previous LRP research and reinforces the idea of weaker neural recruitment of motor preparation resources during imagined rehearsal. The P3 amplitudes obtained in go trials were also smaller for imagined than for real hand movements. Given that the P3 amplitude has been related to the level of attention (Kok, 2001) or motivational aspects (Carrillo-de-laPeña \& Cadaveira, 2000), overall our results suggest that real performance may be associated with greater engagement of top-down mechanisms than is imagined motor execution.

The reduction in mu and beta power during motor imagery has been used as a communicating system in many braincomputer interfaces (McFarland et al., 2000). Our findings suggest that the indices of inhibition obtained in motor imagery may also serve as communicating systems, thus enabling development of more sophisticated brain-computer interfaces.

Despite the contributions above, there are several limitations to our experimental design. As we stated in the Method section, the order of tasks was not counterbalanced. Although this procedure could induce order-related effects, we preferred the participants to have recent experience performing the stopsignal task, because this should facilitate more vivid mental rehearsal. Another limitation is that the SSD was fixed in the imagined task and variable in the real task (following the stair tracking algorithm). Although it could be argued that the stop signal could be easily predicted in the imagined condition, the random presentation of the stop sound on $30 \%$ of the trials and the engagement of processing resources to the performance of the bimanual task made this unlikely. It also seems that millisecond time differences in multisensory stimulisuch as the visual and auditory information used here - are quite difficult to perceive by humans (see Spence \& Squire, 2003). Finally, there are some limitations to the stop-go correction. Although similar procedures have been applied in numerous previous studies, this method is potentially limited by the possible induction of spurious activity in the corrected stop ERPs. This limitation may be more important in SS trials with no behavioral response. Motor-related activity from go trials - inverted in polarity - may be introduced in the SS condition due to the subtraction.

\section{Conclusion}

The high temporal resolution of ERPs and the methodology applied here enabled us to isolate the neural activity associated with the inhibition process, separating it from the processes triggered by the previous go signal. The ERP data were complemented with a time-frequency analysis, to provide additional information about non-phase-locked oscillatory activity.

The exogenous ERPs were similar in the real and imagined tasks, which suggests that participants attended go and stop signals in a similar ways in both conditions.

Imagined performance of the stop-signal task produced stop-N2 and stop-P3 waves and midfrontal theta power changes similar than those observed during overt performance. The similar patterns of brain electrical activity across conditions suggest that inhibition can be trained when overt motor cancellation is not feasible, which opens the possibility of using neural inhibitory activity as a communicating signal in braincomputer interface devices.

Imagined motor execution was also associated with motor $\mathrm{mu}$ and beta oscillations, although with smaller power decreases than in real performance, supporting previous reports of weaker activation of motor cortex during mental rehearsal, and also with smaller P3b amplitudes.

Altogether, our findings suggest that imagined performance of a stop-signal task produces a similar pattern of brain electrical activity to that associated with real performance of the task, although with diminished amplitude. This may be explained by several factors, such as weaker neural recruitment of motor areas, and probably reduced processing of erroneous execution during imagery. Manipulations that increase top-down attention and provide feedback- or errordetection-like rewards during mental rehearsal could diminish the differences in brain activity between real and imagined execution.

Author note A.G.-V. was supported by a grant from the Fundación Ramón Dominguez. The authors thank Fermín Pintos for his support in developing a script for extracting the behavioral data.

\section{References}

Alves, H., Voss, M. W., Boot, W. R., Deslandes, A., Cossich, V., Salles, J. I., \& Krämer, A. F. (2013). Perceptual-cognitive expertise in elite volleyball players. Frontiers in Psychology, 4, 36. doi: $10.3389 /$ fpsyg. 2013.00036

Aron, A. R. (2011). From reactive to proactive and selective control: Developing a richer model for stopping inappropriate responses. Biological Psychiatry, 69, e55-e68. doi:10.1016/j. biopsych.2010.07.024

Band, G. P., \& van Boxtel, G. J. (1999). Inhibitory motor control in stop paradigms: Review and reinterpretation of neural mechanisms. Acta Psychologica, 101, 179-211.

Band, G. P., van der Molen, M. W., \& Logan, G. D. (2003). Horse-race model simulations of the stop-signal procedure. Acta Psychologica, 112, 105-142.

Bekker, E. M., Kenemans, J. L., Hoeksma, M. R., Talsma, D., \& Verbaten, M. N. (2005). The pure electrophysiology of stopping. International Journal of Psychophysiology, 55, 191-198. 
Botvinick, M. M., Cohen, J. D., \& Carter, C. S. (2004). Conflict monitoring and anterior cingulate cortex: An update. Trends in Cognitive Sciences, 8, 539-546. doi:10.1016/j.tics.2004.10.003

Braver, T. S., Barch, D. M., Gray, J. R., Molfese, D. L., \& Snyder, A. (2001). Anterior cingulate cortex and response conflict: Effects of frequency, inhibition and errors. Cerebral Cortex, 11, 825-836.

Carrillo-de-la-Peña, M. T., \& Cadaveira, F. (2000). The effect of motivational instructions on P300 amplitude. Clinical Neurophysiology, 30, 232-239.

Carrillo-de-la-Peña, M. T., Galdo-Álvarez, S., \& Lastra-Barreira, C. (2008). Equivalent is not equal: Primary motor cortex (MI) activation during motor imagery and execution of sequential movements. Brain Research, 1226, 134-143. doi:10.1016/j.brainres.2008.05.089

Carrillo-de-la-Peña, M. T., Lastra-Barreira, C., \& Galdo-Álvarez, S. (2006). Limb (hand vs. foot) and response conflict have similar effects on event-related potentials (ERPs) recorded during motor imagery and overt execution. European Journal of Neuroscience, 24, 635-643. doi:10.1111/j.1460-9568.2006.04926.x

Cavanagh, J. F., \& Frank, M. J. (2014). Frontal theta as a mechanism for cognitive control. Trends in Cognitive Sciences, 18, 414-421. doi: 10.1016/j.tics.2014.04.012

Cavanagh, J. F., Zambrano-Vazquez, L., \& Allen, J. J. B. (2012). Theta lingua franca: A common mid-frontal substrate for action monitoring processes. Psychophysiology, 49, 220-238. doi:10.1111/j.14698986.2011.01293.x

Chamberlain, S., Fineberg, N., Blackwell, A., Robbins, T., \& Sahakian, B. (2006). Motor inhibition and cognitive flexibility in obsessivecompulsive disorder and trichotillomania. American Journal of Psychiatry, 163, 1282-1284.

Cisek, P., \& Kalaska, J. F. (2004). Neural correlates of mental rehearsal in dorsal premotor cortex. Nature, 431, 993-996.

Dehaene, S., Posner, M. I., \& Tucker, D. M. (1994). Localization of a neural system for error detection and compensation. Psychological Science, 5, 303-305. doi:10.1111/j.1467-9280.1994.tb00630.x

Delorme, A., \& Makeig, S. (2004). EEGLAB: An open source toolbox for analysis of single-trial EEG dynamics including independent component analysis. Journal of Neuroscience Methods, 134, 9-21. doi:10.1016/j.jneumeth.2003.10.009

Dien, J. (2010). The ERP PCA Toolkit: An open source program for advanced statistical analysis of event-related potential data. Journal of Neuroscience Methods, 187, 138-145.

Dien, J., \& Frishkoff, G. A. (2005). Introduction to principal components analysis of event-related potentials. In T. Handy (Ed.), Event-related potentials: A methods handbook (pp. 137-153). Cambridge, MA: MIT Press.

Dimoska, A., \& Johnstone, S. J. (2007). Neural mechanisms underlying trait impulsivity in non-clinical adults: Stop-signal performance and event-related potentials. Progress in Neuro-Psychopharmacology \& Biological Psychiatry, 31, 443-454.

Dimoska, A., Johnstone, S. J., \& Barry, R. J. (2006). The auditory-evoked $\mathrm{N} 2$ and P3 components in the stop-signal task: Indices of inhibition, response-conflict or error-detection? Brain and Cognition, 62, 98112. doi:10.1016/j.bandc.2006.03.011

Driskell, J. E., Copper, C., \& Moran, A. (1994). Does mental practice enhance performance? Journal of Applied Psychology, 79, 481-492.

Enriquez-Geppert, S., Konrad, C., Pantev, C., \& Huster, R. J. (2010). Conflict and inhibition differentially affect the N200/P300 complex in a combined go/nogo and stop-signal task. Neurolmage, 51, 877887.

Galdo-Álvarez, S., \& Carrillo-de-la-Peña, M. T. (2004). ERP evidence of MI activation without motor response execution. NeuroReport, 15, 2067-2070.

Grèzes, J., \& Decety, J. (2001). Functional anatomy of execution, mental simulation, observation, and verb generation of actions: A metaanalysis. Human Brain Mapping, 12, 1-19. doi:10.1002/10970193(200101)12:1<1::AID-HBM10>3.0.CO;2-V
Harmony, T., Alba, A., Marroquín, J. L., \& González-Frankenberger, B. (2009). Time-frequency-topographic analysis of induced power and synchrony of EEG signals during a Go/No-Go task. International Journal of Psychophysiology, 71, 9-16.

Harper, J., Malone, S. M., \& Bernat, E. M. (2014). Theta and delta band activity explain N2 and P3 ERP component activity in a go/no-go task. Clinical Neurophysiology, 125, 124-132.

Hughes, M. E., Fulham, W. R., Johnston, P. J., \& Michie, P. T. (2012). Stop-signal response inhibition in schizophrenia: Behavioural, event-related potential and functional neuroimaging data. Biological Psychology, 89, 220-231.

Huster, R. J., Enriquez-Geppert, S., Lavallee, C. F., Falkenstein, M., \& Herrmann, C. S. (2013). Electroencephalography of response inhibition tasks: Functional networks and cognitive contributions. International Journal of Psychophysiology, 87, 217-233.

Huster, R. J., Plis, S. M., Lavallee, C. F., Calhoun, V. D., \& Herrmann, C. S. (2014). Functional and effective connectivity of stopping. Neuroimage, 94, 120-128.

Huster, R. J., Westerhausen, R., Pantev, C., \& Konrad, C. (2010). The role of the cingulate cortex as neural generator of the N200 and P300 in a tactile response inhibition task. Human Brain Mapping, 31, 12601271. doi:10.1002/hbm. 20933

Jeannerod, M., \& Frak, V. (1999). Mental imaging of motor activity in humans. Current Opinion in Neurobiology, 9, 735-739.

Kayser, J., \& Tenke, C. E. (2006). Principal components analysis of Laplacian waveforms as a generic method for identifying ERP generator patterns: II. Adequacy of low-density estimates. Clinical Neurophysiology, 117, 369-380.

Kirmizi-Alsan, E., Bayraktaroglu, Z., Gurvit, H., Keskin, Y. H., Emre, M., \& Demiralp, T. (2006). Comparative analysis of event-related potentials during Go/NoGo and CPT: Decomposition of electrophysiological markers of response inhibition and sustained attention. Brain Research, 1104, 114-128. doi:10.1016/j.brainres.2006.03.010

Kok, A. (2001). On the utility of $\mathrm{P} 3$ amplitude as a measure of processing capacity. Psychophysiology, 38, 557-577.

Kok, A., Ramautar, J. R., De Ruiter, M. B., Band, G. P. H., \& Ridderinkhof, K. R. (2004). ERP components associated with successful and unsuccessful stopping in a stop-signal task. Psychophysiology, 41, 9-20. doi:10.1046/j.1469-8986. 2003.00127.x

Krämer, U. M., Knight, R. T., \& Münte, T. F. (2011). Electrophysiological evidence for different inhibitory mechanisms when stopping or changing a planned response. Journal of Cognitive Neuroscience, 23, 2481-2493.

Krämer, U. M., Solbakk, A.-K., Funderud, I., Løvstad, M., Endestad, T., \& Knight, R. T. (2013). The role of the lateral prefrontal cortex in inhibitory motor control. Cortex, 49, 837-849.

Kranczioch, C., Mathews, S., Dean, P. J., \& Sterr, A. (2009). On the equivalence of executed and imagined movements: Evidence from lateralized motor and nonmotor potentials. Human Brain Mapping, 30, 3275-3286.

Lansbergen, M. M., Böcker, K. B., Bekker, E. M., \& Kenemans, J. L. (2007). Neural correlates of stopping and self-reported impulsivity. Clinical Neurophysiology, 118, 2089-2103.

Lawrence, A. J., Luty, J., Bogdan, N. A., Sahakian, B. J., \& Clark, L. (2009). Impulsivity and response inhibition in alcohol dependence and problem gambling. Psychopharmacology, 207, 163-172. doi: 10.1007/s00213-009-1645-x

Lijffijt, M., Kenemans, J. L., Verbaten, M. N., \& van Engeland, H. (2005). A meta-analytic review of stopping performance in attention-deficit/hyperactivity disorder: Deficient inhibitory motor control? Journal of Abnormal Psychology, 114, 216-222.

Logan, G. D. (1994). On the ability to inhibit thought and action: A users' guide to the stop signal paradigm. In D. Dagenbach \& T. H. Carr (Eds.), Inhibitory processes in attention, memory and language (pp. 189-239). New York, NY: Academic Press. 
Logan, G. D., \& Cowan, W. B. (1984). On the ability to inhibit thought and action: A theory of an act of control. Psychological Review, 91, 295-327. doi:10.1037/0033-295X.91.3.295

Luu, P., Tucker, D. M., \& Makeig, S. (2004). Frontal midline theta and the error-related negativity: Neurophysiological mechanisms of action regulation. Clinical Neurophysiology, 115, 1821-1835.

McFarland, D. J., Miner, L. A., Vaughan, T. M., \& Wolpaw, J. R. (2000). $\mathrm{Mu}$ and beta rhythm topographies during motor imagery and actual movements. Brain Topography, 12, 177-186.

Müller, V., \& Anokhin, A. P. (2012). Neural synchrony during response production and inhibition. PLoS ONE, 7, e38931. doi:10.1371/ journal.pone.0038931

Nieuwenhuis, S., Yeung, N., van den Wildenberg, W., \& Ridderinkhof, K. R. (2003). Electrophysiological correlates of anterior cingulate function in a go/no-go task: Effects of response conflict and trial type frequency. Cognitive, Affective, \& Behavioral Neuroscience, 3, 17-26. doi:10.3758/CABN.3.1.17

Pfurtscheller, G., Brunner, C., Schlögl, A., \& Lopes da Silva, F. H. (2006). Mu rhythm (de)synchronization and EEG single-trial classification of different motor imagery tasks. NeuroImage, 31, 153159. doi:10.1016/j.neuroimage.2005.12.003

Pfurtscheller, G., \& Neuper, C. (1997). Motor imagery activates primary sensorimotor area in humans. Neuroscience Letters, 239, 65-68.

Pfurtscheller, G., Neuper, C., Brunner, C., \& da Silva, F. L. (2005). Beta rebound after different types of motor imagery in man. Neuroscience Letters, 378, 156-159.

Pliszka, S. R., Liotti, M., \& Woldorff, M. G. (2000). Inhibitory control in children with attention-deficit/hyperactivity disorder: Event-related potentials identify the processing component and timing of an impaired right-frontal response-inhibition mechanism. Biological Psychiatry, 48, 238-246.

Ramautar, J. R., Kok, A., \& Ridderinkhof, K. R. (2006). Effects of stopsignal modality on the N2/P3 complex elicited in the stop-signal paradigm. Biological Psychology, 72, 96-109.

Randall, W. M., \& Smith, J. L. (2011). Conflict and inhibition in the cuedGo/NoGo task. Clinical Neurophysiology, 122, 2400-2407.

Rubia, K., Smith, A. B., Taylor, E., \& Brammer, M. (2007). Linear agecorrelated functional development of right inferior fronto-striatocerebellar networks during response inhibition and anterior cingulate during error-related processes. Human Brain Mapping, 28, 1163-1177. doi:10.1002/hbm.20347

Schall, J. D., Stuphorn, V., \& Brown, J. W. (2002). Monitoring and control of action by the frontal lobes. Neuron, 36, 309-322.

Schmajuk, M., Liotti, M., Busse, L., \& Woldorff, M. G. (2006). Electrophysiological activity underlying inhibitory control processes in normal adults. Neuropsychologia, 44, 384-395. doi:10.1016/j.neuropsychologia.2005.06.005

Schmiedt-Fehr, C., \& Basar-Eroglu, C. (2011). Event-related delta and theta brain oscillations reflect age-related changes in both a general and a specific neuronal inhibitory mechanism. Clinical Neurophysiology, 122, 1156-1167.

Shen, I.-H., Lee, D.-S., \& Chen, C.-L. (2014). The role of trait impulsivity in response inhibition: Event-related potentials in a stopsignal task. International Journal of Psychophysiology, 91, 80-87. doi:10.1016/j.ijpsycho.2013.11.004

Spence, C., \& Squire, S. (2003). Multisensory integration: Maintaining the perception of synchrony. Current Biology, 13, R519-R521.

Stavrinou, M. L., Moraru, L., Cimponeriu, L., Della Penna, S., \& Bezerianos, A. (2007). Evaluation of cortical connectivity during real and imagined rhythmic finger tapping. Brain Topography, 19, 137-145.

van den Wildenberg, W. P., van der Molen, M. W., \& Logan, G. D. (2002). Reduced response readiness delays stop signal inhibition. Acta Psychologica, 111, 155-169.

Verbruggen, F., \& Logan, G. D. (2009). Models of response inhibition in the stop-signal and stop-change paradigms. Neuroscience \& Biobehavioral Reviews, 33, 647-661. doi:10.1016/j.neubiorev. 2008.08.014

Verburgh, L., Scherder, E. J., van Lange, P. A., \& Oosterlaan, J. (2014). Executive functioning in highly talented soccer players. PLOS ONE, 9, e91254.

Wang, C. H., Chang, C. C., Liang, Y. M., Shih, C. M., Chiu, W. S., Tseng, P.,...Juan, C. H. (2013). Open vs. closed skill sports and the modulation of inhibitory control. PLoS ONE, 8, e55773. doi:10.1371/ journal.pone.0055773

Wessel, J. R., \& Aron, A. R. (2015). It's not too late: The onset of the frontocentral P3 indexes successful response inhibition in the stopsignal paradigm. Psychophysiology, 52, 472-480. 